\title{
Large Electric-Field Modulation of Magnetic Properties in Fe Films on $\mathrm{BiScO}_{3}-\mathrm{PbTiO}_{3}$ Ceramics
}

\author{
Jing Wang, Han Wang, He Jiang, Xiaohui Wang, Yuanhua Lin, and C. W. Nan \\ Department of Materials Science and Engineering, State Key Lab of New Ceramics and Fine Processing, Tsinghua University, \\ Beijing 100084, China \\ Correspondence should be addressed to C.W. Nan, cwnan@tsinghua.edu.cn
}

Received 13 December 2009; Accepted 20 January 2010

Academic Editor: Christian Brosseau

Copyright ( 92010 Jing Wang et al. This is an open access article distributed under the Creative Commons Attribution License, which permits unrestricted use, distribution, and reproduction in any medium, provided the original work is properly cited.

Based on the magneto-optical Kerr effect, we report the electric-field modulation of the magnetic properties in $\mathrm{Fe} / \mathrm{BiScO}{ }_{3}-\mathrm{PbTiO} 3$ (BSPT) film-on-ceramic substrate structure. The Fe films are directly grown on the fully-poled BSPT ceramic substrates by magnetron sputtering. An electric field applied parallel to the prepolarization direction of the piezoelectric BSPT can induce a reversible increase in the coercive field $H_{c}$ of about $30 \%$, whereas an electric field antiparallel to the prepolarization direction can cause a persistent, tremendous decrease (as large as 97\%) in $H_{c}$, and a small reversal electric field can resume it back. The strain induced by the inverse piezoelectric effect is the primary mechanism behind. This large modulation of the coercive field by the electric field could inspire further exploration of electric-field-controlled magnetic switching in multiferroic heterostructures.

\section{Introduction}

Recently, increasing attention has been focused on multiferroic materials which simultaneously exhibit two or more properties of ferroelectricity, (anti-)ferromagnetism, and ferroelasticity [1]. Of the multiferroics family, magnetoelectric (ME) composites, especially nano-ME-composites [2], are of interest due to their great scientific and technological importance. These composite ME materials not only have both ferroelectric (FE) and (anti-)ferromagnetic (FM) orderings but also show a large ME coupling effect at room temperature (i.e., a polarization induced by an external magnetic field or a magnetization induced by an applied voltage) [3-5]. Thus ME composites make it possible to control the FM (FE) ordering via electric (magnetic) field at room temperature and might lead to the next-generation of memories, sensors, generators and transducers, and so forth.

Up to now, magnetic-field control of electric polarization in ME composites has been widely reported [5]. Lately, as the magnetic random access memory (MRAM) becomes more and more popular, electric field control of the magnetism, which can be easily achieved in the ME composites at room temperature, has attracted ever growing attention and been the subject of intensive investigations. Electric control of magnetism would replace the traditional magnetic field control induced by the electromagnets which are slow, heavy, and power-consumed and take the advantages of fast control, miniaturization, and power-efficiency.

Manipulating magnetization or the magnetic anisotropy directly by using an electric field rather than the spinpolarized currents has become an important issue in the field of the ME materials [6]. Several groups have made their effort to investigate such manipulation in some systems (e.g., epitaxial $\mathrm{BiFeO}_{3}-\mathrm{CoFe}_{2} \mathrm{O}_{4}$ columnar nanostructures, $\mathrm{Fe}_{3} \mathrm{O}_{4} / \mathrm{Pb}(\mathrm{Zr}, \mathrm{Ti}) \mathrm{O}_{3}$ and $\mathrm{Co}_{50} \mathrm{Fe}_{50} / \mathrm{Pb}(\mathrm{Zr}, \mathrm{Ti}) \mathrm{O}_{3}$ hybrid structures) by using different methods (e.g., magnetic-force microscopy, ferromagnetic resonance, and magneto-optical Kerr effect (MOKE) magnetometer) [7-9]. In these works the maximum change in the magnetic coercive field at room temperature is about $80 \%$ at an electric field as high as $75 \mathrm{kV} / \mathrm{cm}$, observed in the patterned $\mathrm{Co}_{50} \mathrm{Fe}_{50}$ film on the $\mathrm{Pb}(\mathrm{Zr}, \mathrm{Ti}) \mathrm{O}_{3}$ substrate [9]. Even though a coercive field change of up to $120 \%$ was observed in the $\mathrm{Fe} / \mathrm{BaTiO}_{3}$ heterostructures, it only appeared at the $\mathrm{R} \rightarrow \mathrm{O}$ (rhombohedral to orthorhombic phase) transition at about $190 \mathrm{~K}$, far below the room temperature [10]. In this paper, by means of the room-temperature MOKE measurement, we present a significant electric-field control of the magnetic properties 
of Fe films directly grown on $\mathrm{BiScO}_{3}-\mathrm{PbTiO}_{3}$ substrate via the ME coupling. For example, a large change in the coercive field $H_{c}$, that is, $\Delta H_{c}=\left(H_{c}(E)-H_{c}(0)\right) / H_{c}(0)$, of the Fe films of $-97 \%$ was observed at an applied electric field of about $-15 \mathrm{kV} / \mathrm{cm}$, and our observation also demonstrates interesting switchable electric-field-controlled magnetic behavior in the hybrid structures.

\section{Experimental}

$0.37 \mathrm{BiScO}_{3}-0.63 \mathrm{PbTiO}_{3}$ (BSPT) ceramics was chosen as the ferroelectric substrate because of its high piezoelectric coefficient [11], relatively high density by using two-step sintering technique [12], and low cost compared with the single crystal substrates [13], as well. The nanopowder used to acquire the highly dense BSPT ceramics was synthesized by the citrate sol-gel method. After the ball milling and drying, the powder was pressed into pellets with a diameter of $10 \mathrm{~mm}$ and a thickness of about $1 \mathrm{~mm}$ under a uniaxial pressure of $3 \mathrm{MPa}$. The green pellets were then first heated to a higher temperature ranging from $1000^{\circ} \mathrm{C}$ to $1050^{\circ} \mathrm{C}$ with a heating rate of about $4^{\circ} \mathrm{C} / \mathrm{min}$, then immediately cooled to a lower temperature of about $800^{\circ} \mathrm{C}$ with a cooling rate of about $20^{\circ} \mathrm{C} / \mathrm{min}$, and finally held at $800^{\circ} \mathrm{C}$ for about $4 \sim 8$ hours. The processing details were described elsewhere [12]. The sintered BSPT ceramics was fully poled along the thickness direction under an electric field of $40 \mathrm{kV} / \mathrm{cm}$ for 15 minutes in a silicon oil bath at about $140^{\circ} \mathrm{C}$.

Consequently, the fully poled BSPT ceramics was grinded into a thickness of about $0.6 \mathrm{~mm}$ and then polished to ensure the low surface roughness as substrates. The Fe film was then deposited on the cleaned BSPT substrate by radio frequency magnetron sputtering at room temperature. Two germanium (Ge, 99.9\%) strips were attached on the iron (Fe, 99.9\%) target to accelerate the deposition and adjust the Ge content in the Fe film. The base pressure of the sputtering chamber was about $8 \times 10^{-4} \mathrm{~Pa}$, and the working pressure was $1.0 \mathrm{~Pa}$ of argon. The sputtering power was kept at $180 \mathrm{~W}$ (the corresponding deposition rate is about $2.5 \mathrm{~nm} / \mathrm{min}$ ) during the whole deposition of 180 minutes.

X-ray diffraction (XRD, RIGAKA D/max-RB) with $\mathrm{Cu}$ $\mathrm{K} \alpha$ radiation, the atomic force microscopy (AFM, PISA XEI100), and the X-ray fluorescence (XRF, SHIMADZU XRF-1800) were used for the phase analysis, the surface morphology and roughness analysis, and the content determination of Ge in the Fe film, respectively. The polarizationelectric field $(P-E)$ hysteresis loop of the BSPT was measured by using the ferroelectric test system (Radiant Tech. Inc., RT 609B) at $10 \mathrm{~Hz}$ and $1 \mathrm{~Hz}$. The magneto-optical Kerr effect magnetometer (Nano MOKE 2, Durham Magneto Optics Ltd.) was used to characterize the electric field control of the magnetic properties of the Fe films. All the measurements were performed at room temperature.

\section{Results and Discussion}

Figure $1(\mathrm{a})$ is the X-ray diffraction pattern of the BSPT ceramics. The splitting peaks of $\{100\}$ and $\{200\}$ implicit the

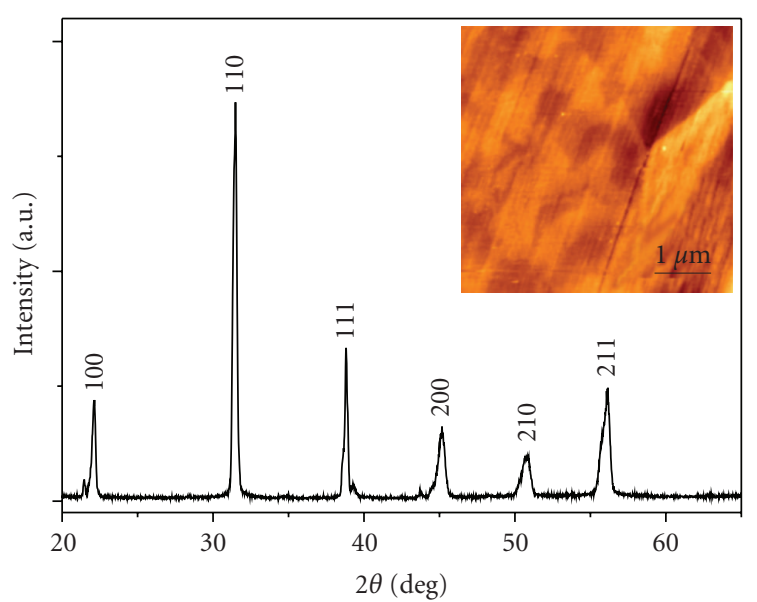

(a)

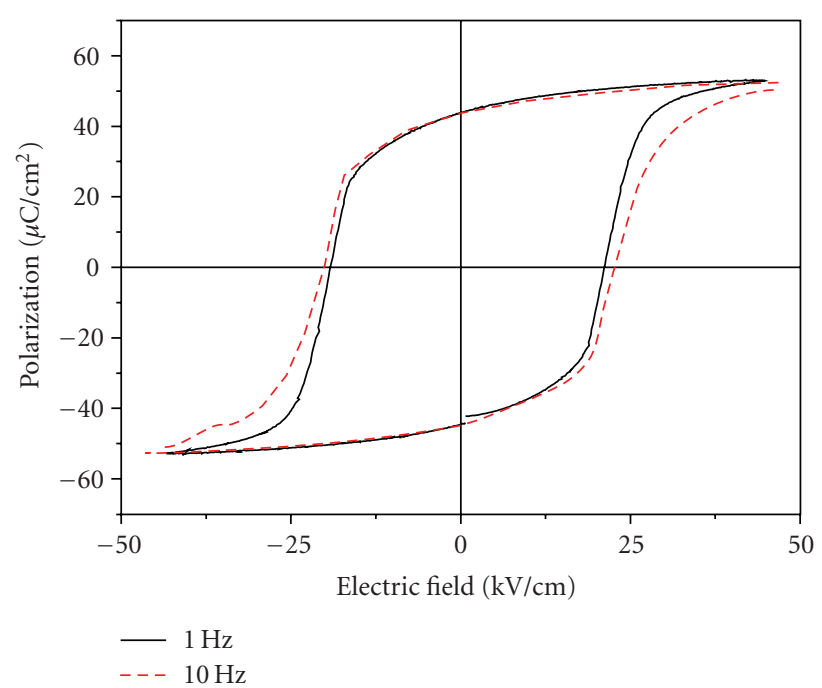

(b)

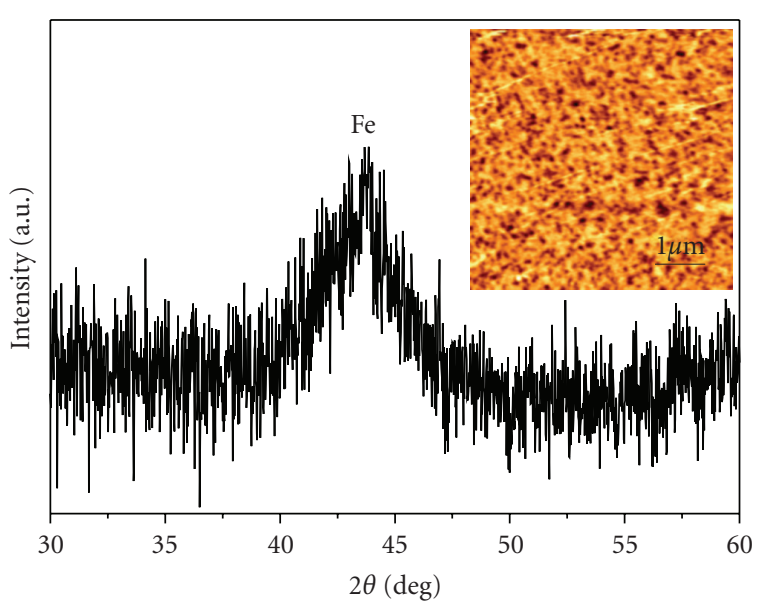

(c)

Figure 1: (a) X-ray diffraction (XRD) pattern and (b) the polarization-electric field loops of the BSPT ceramics. (c) XRD pattern of the Fe films. The insets of (a) and (c) are the AFM images of the polished BSPT ceramic surface and the Fe film, respectively. 


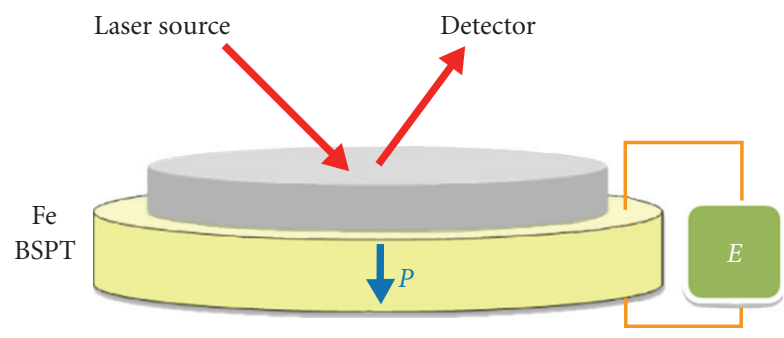

(a)

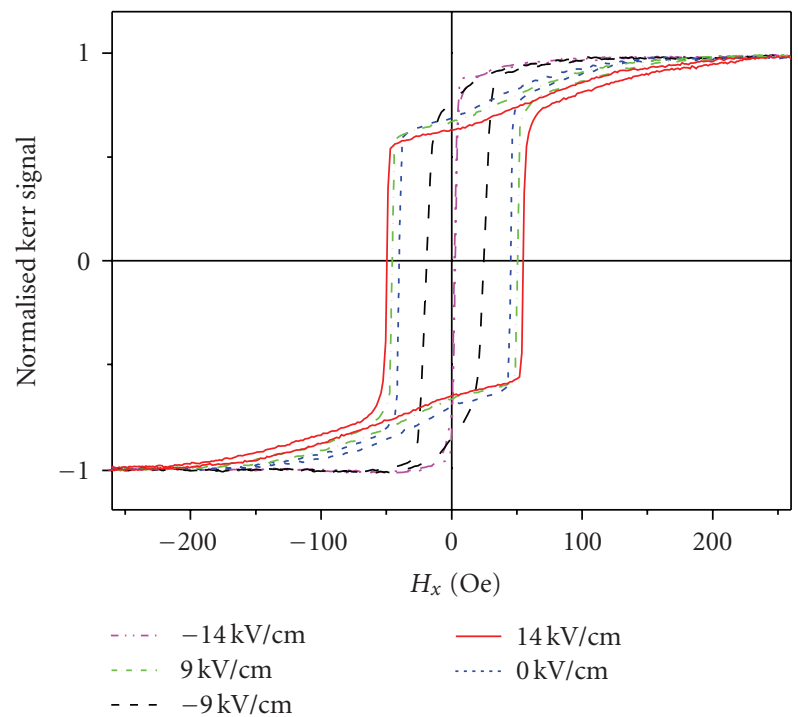

(b)

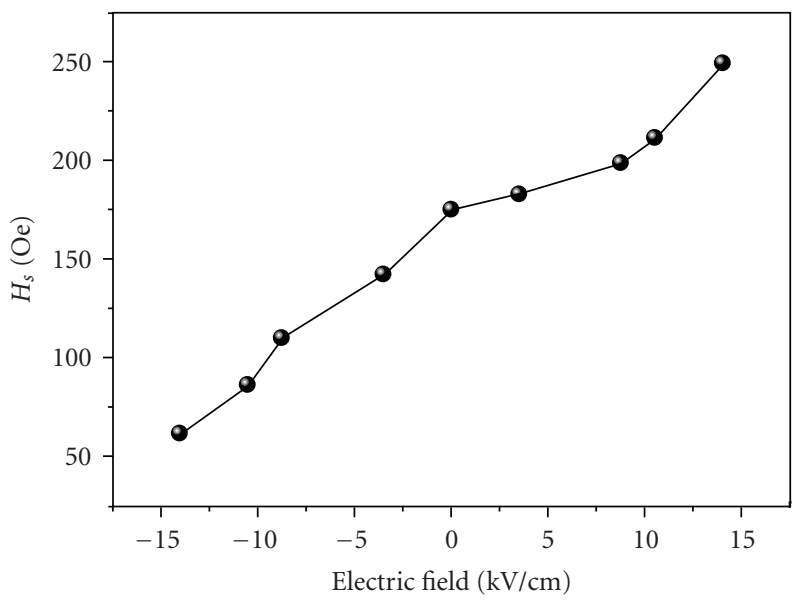

(c)

Figure 2: (a) Schematic of the MOKE measurement, (b) the normalized MOKE hysteresis loops, and (c) the saturated magnetic field $H_{s}$ of the sample under different electric fields. $P$ and $E$ in (a) denote the prepolarization vector and the external electric field, respectively.

morphotropic phase boundary of $(1-x) \mathrm{BiScO}_{3}-x \mathrm{PbTiO}_{3}$ system at $x=0.63[11,12]$. Figure 1(b) exhibits the $P-E$ loops of the BSPT ceramics measured in the silicon oil bath at room temperature. Obviously, the loops got saturated at about $45 \mathrm{kV} / \mathrm{cm}$. The remnant polarization $\left(P_{r}\right)$ and conceive field $\left(E_{c}\right)$ are about $44 \mu \mathrm{C} / \mathrm{cm}^{2}$ and $22 \mathrm{kV} / \mathrm{cm}$, respectively. Also, the atomic force microscopy of the polished BSPT ceramics (inset of Figure 1(a)) demonstrates the relatively smooth surface with the root-mean-square roughness of less than $1.3 \mathrm{~nm}$ in the range of $5 \times 5 \mu \mathrm{m}^{2}$, which ensures the following deposition of Fe films. Since the whole deposition was performed at room temperature, the Fe film was basically amorphous, as demonstrated by the XRD (Figure 1(c)). The AFM image of the Fe film showing no obvious grains (inset of Figure 1(c)) also reveals the amorphous phase of the Febased alloy. The thickness of the Fe film is about $420 \mathrm{~nm}$. The content of Ge in the Fe film, measured by the X-ray Fluorescence, is about 10.0 atom $\%$.

Since the vibration plane of the linearly polarized light changes when it reflects from the surface of the magnetized medium (i.e., MOKE effect), we can directly characterize the magnetic behavior of magnetic films by using the MOKE magnetometer. The Kerr signal, which is proportional to the magnetization, can be used to probe the local magnetic prop- erties of the Fe films. In our measurement (see Figure 2(a)), direct-current voltages were applied upon the BSPT substrate along its thickness direction by using a voltage sourcemeter (Keithley 2410). Electric voltages ranging from $-1100 \mathrm{~V}$ to $1100 \mathrm{~V}$ (i.e., the utmost limit acquired by Keithley 2410) were applied on the fully polarized BSPT substrate (corresponding to a maximum electric field of $-19 \mathrm{kV} / \mathrm{cm}$ to $+19 \mathrm{kV} / \mathrm{cm}$ on the substrate), and the longitudinal Kerr signals were measured to conclude the electric field control of the magnetic properties.

Figure 2(b) shows the representative Kerr hysteresis loops under different electric fields such as positive electric fields (i.e., the applied electric field parallel to the prepolarization direction of the BSPT, as shown in Figure 2(a)) and negative ones (i.e. the applied electric field antiparallel to the prepolarization direction). Obvious changes in the Kerr hysteresis loops occur upon the electric fields. The hysteresis loop without voltage bias is almost squared and the coercive field $H_{c}$ is about $42 \mathrm{Oe}$. Upon the positive electric fields, the loops become a little wider and retain similar shapes. For example, the coercive field increases up to 51 Oe when the electric field is up to $14 \mathrm{kV} / \mathrm{cm}$. Of interest to note is that not only the obvious decreases in the coercive fields but also significant shape changes are observed when the 
negative electric fields are applied on the BSPT. The hysteresis loops become more narrow and narrow with the increase in the absolute value of negative electric fields. For example, upon an electric field of $-14 \mathrm{kV} / \mathrm{cm}$, the loop becomes a very slim hysteresis one with a coercive field of $1.3 \mathrm{Oe}$, which is only about $3 \%$ of 42 Oe at zero electric field. In other words, just upon an electric field of $-14 \mathrm{kV} / \mathrm{cm}$ at room temperature, as high as $97 \%$ decrease in the coercive field occurs. The magnetic free energy of the films accounts for this. In films, the demagnetization energy favors an inplane easy axis, which is parallel to the external magnetic field direction in the isotropic amorphous films. Since the BSPT is prepolarized, a positive (negative) voltage along the thickness direction will lead to the compressive (tensile) strain along the radical direction. This deformation is then directly transferred to the Fe film, yielding the compressive (tensile) strain in the film, as well. This compressive (tensile) strain resists (promotes) the easy axis along the in-plane direction [14] due to its positive magnetostrictive coefficient $\lambda$ of the amorphous Fe films [15]. Hence, the increase (decrease) in the coercive field $H_{c}$ is observed. Besides $H_{c}$, the changes in the saturated magnetic field $H_{s}$ (i.e., the magnetic field at which the magnetization gets saturated) can also demonstrate the above explanation (see Figure 2(c)). The MOKE measurements focused at different regions on the Fe film presented similar behavior.

Detailed changes in the magnetic coercive fields with the electric field $E$ are presented in Figure 3 . The change in the coercive field $\Delta H_{c}$ is used to characterize the modulation of the electric field. When the positive electric field is applied on the sample, $H_{c}$ of the Fe film increases linearly with increasing $E$ (see the arrow (1)). During the following process of $E$ gradually decreasing from $19 \mathrm{kV} / \mathrm{cm}$ down to zero, $H_{c}$ follows the same path (see the arrow (2)) to the original at zero bias. As shown, when $E$ is positive, $\Delta H_{c}$ slowly decreases as $E$ approaches to zero and is proportional to the electric field. However, when the electric field further decreases to the negative side, totally different change in the coercive field occurs. As shown in Figure 3(a), when $E$ is negative and its absolute value increases, $\Delta H_{c}$ decreases dramatically down to about 1 Oe at $E=-15 \mathrm{kV} / \mathrm{cm}$. The further decrease in the negative electric field does not affect the hysteresis loops very much but retains them in the very slim shapes (Figure 3(a)). After reaching the minimum negative electric field, the following increase in the electric field from $-19 \mathrm{kV} / \mathrm{cm}$ to 0 (Figure 3(b)) cannot change the slim hysteresis until $E$ is switched to positive up to $+1.8 \mathrm{kV} / \mathrm{cm}$. After that, as the electric field increases up, the hysteresis loop gets wider and wider again and finally recovers back to the similar squared shape at zero bias (Figure 3(b)).

Further, more interesting behaviors are observed after the external electric field is switched off. Figure 4(a) presents the change in $H_{c}$ after each bias voltage is switched off, that is, $\Delta H_{c}$ measured at each $E$ back to zero. As seen, in the first applied positive bias region (denoted by arrow 1 ), once the positive electric field is switched off, the magnetic hysteresis loop immediately returns to its original state at $E=0$ (see the circle dots in Figure 4(a)), which agrees with

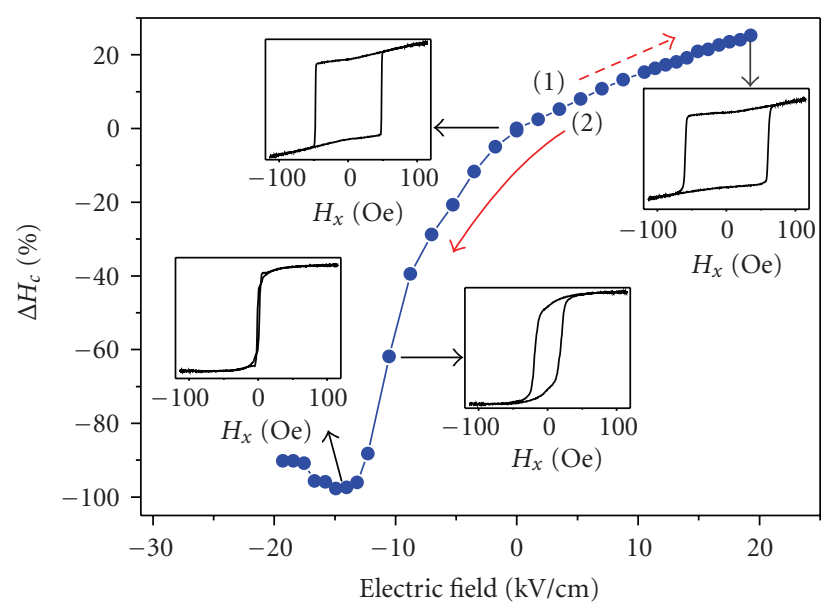

(a)

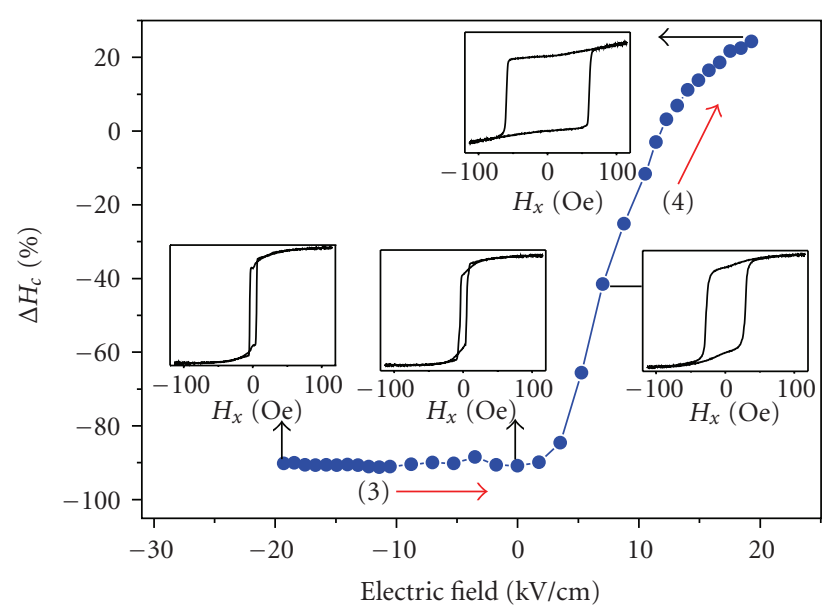

(b)

FIGURE 3: Variations of $H_{c}$ with the electric field decreasing from $19 \mathrm{kV} / \mathrm{cm}$ (a) and increasing up from $-19 \mathrm{kV} / \mathrm{cm}$ (b), respectively. The insets show the representative Kerr hysteresis loops at some electric fields.

Hooke's law in the elastic strain range. Figure 4(b) more clearly demonstrates such a reversible modulation. As an electric field of $+19 \mathrm{kV} / \mathrm{cm}$ is switched on and off alternately, $\Delta H_{c}$ changes between $+30 \%$ and zero immediately. This reversible modulation can be always observed as long as the electric field is parallel to the prepolarization direction of the piezoelectric ceramics, no matter the electric field increases up from zero or decreases to zero.

In contrast to the reversible modulation of the positive electric field, once the negative electric field is applied, the change cannot be completely recovered after the negative electric field is switched off, as shown by the square dots in Figure 4(a). The Fe film, after the negative bias voltage unloaded, keeps the magnetic behavior with it to great extent. In particular, after the negative electric field reaches the minimum one (i.e., $-19 \mathrm{kV} / \mathrm{cm}$ allowed in the present case), $\Delta H_{c}$ measured after this $E$ back to zero remains constant (i.e., about $-92 \%$; see the arrow 3 ) during the 


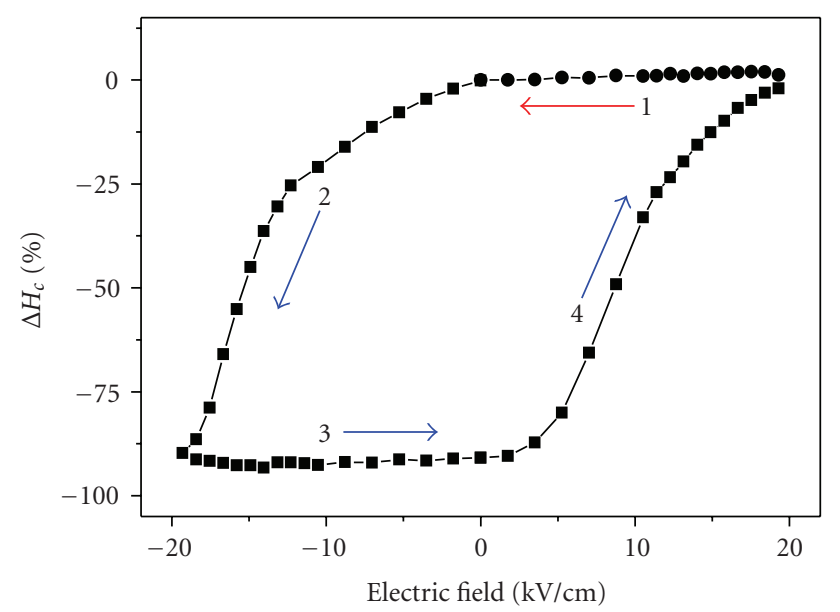

(a)
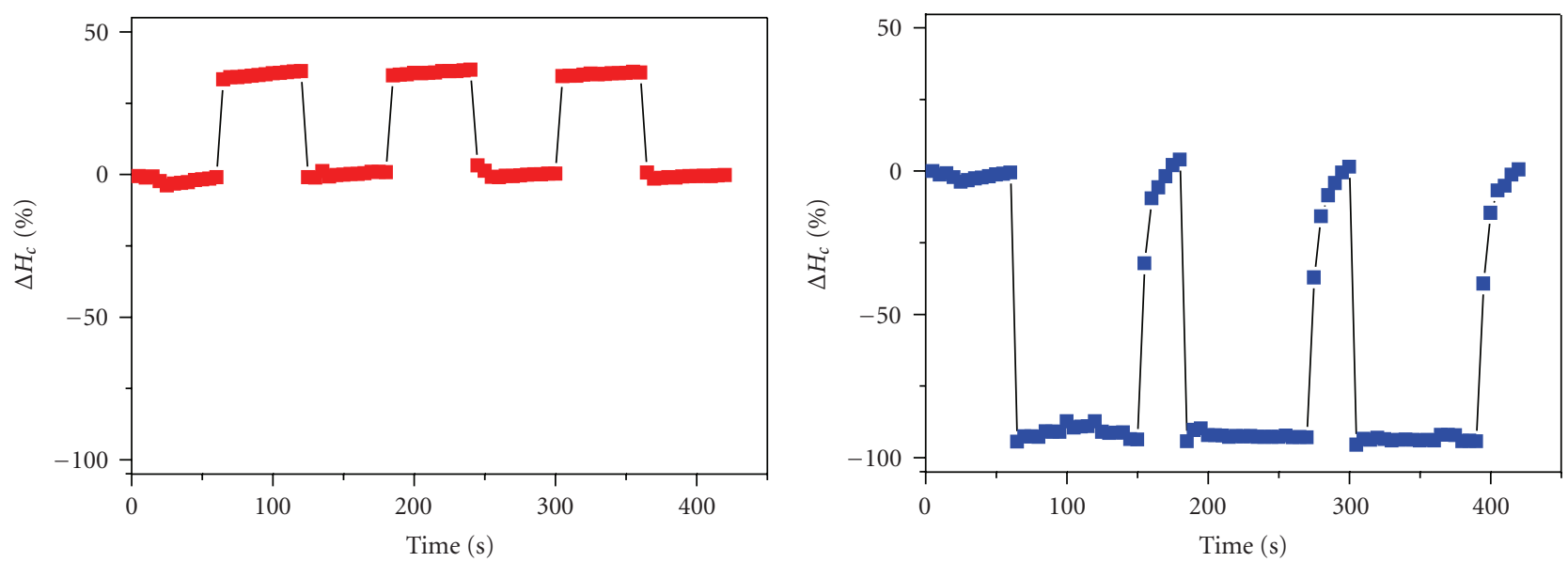

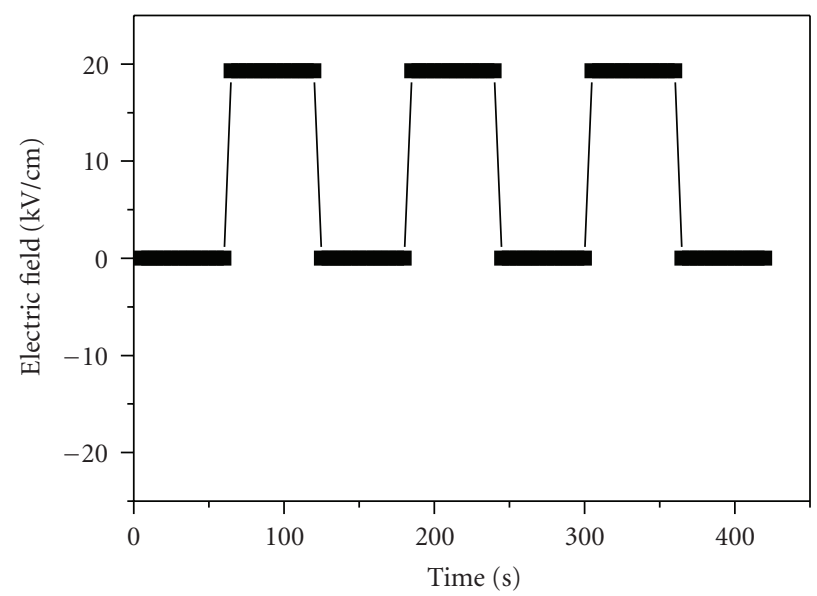

(b)

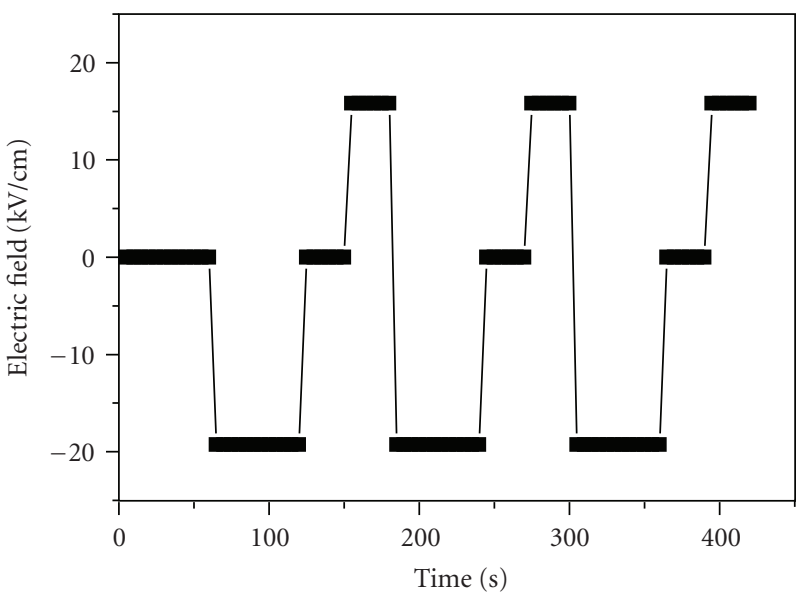

(c)

FIgURE 4: (a) $\Delta H_{c}$ measured after each bias voltage switched off. (b) and (c) are the $\Delta H_{c}$ responses of the Fe/BSPT composites to the electric fields.

following increase from $-19 \mathrm{kV} / \mathrm{cm}$ to zero. Figure $4(\mathrm{c})$ also demonstrates such an irreversible modulation in details. When the electric field of $-19 \mathrm{kV} / \mathrm{cm}$ is switched off, the change in the $H_{c}$ does not recover until a positive electric field of about $+16 \mathrm{kV} / \mathrm{cm}$ triggers it back. Without the trigger electric field, the large $\Delta H_{c}$ stabilizes after the electric field of $-19 \mathrm{kV} / \mathrm{cm}$ is switched off and does not significantly change with time (Figure 5). The small $H_{c}$ due to this unique 


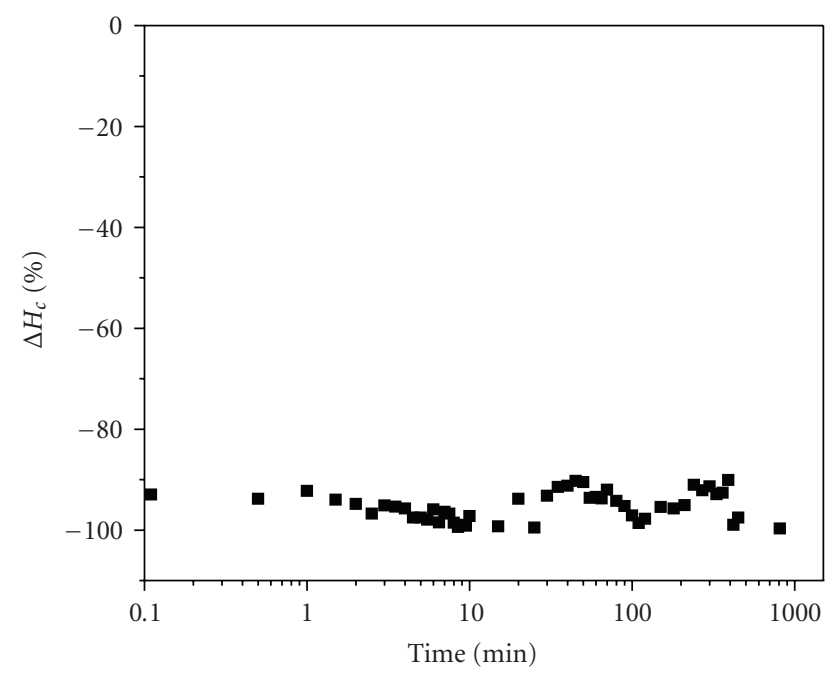

Figure 5: Time dependence of $\Delta H_{c}$ measured after the applied electric field of $-19 \mathrm{kV} / \mathrm{cm}$ is switched off.

memory effect after the unloading of a negative bias voltage can be used to represent the "0-state" in memory devices, while the considerably large $H_{c}$ without any bias voltage can represent the logic "1-state." Both of the large gap between the two platforms in Figure 4 and the time stability of $\Delta H_{c}$ ensure the two definite states of " 0 " and " $1 . "$

\section{Conclusion}

In summary, we have successfully fabricated a simple bilayered hybrid structure by directly sputtering the Fe films on the low-cost BSPT ceramics. Large and reversible electricfield modulations of the magnetic coercive fields, induced by the converse ME coupling effect, are observed at room temperature. Such a magnetic-coercive-field modulation, which can be switched "on" or "off" with comparably small electric fields, would offer a possible way to new ME devices such as memories.

\section{Acknowledgments}

This work was supported by the Ministry of Sciences and Technology of China through the 973-project (Grant no. 2009CB623303) and the National Science Fund of China (Grant nos. 50832003 and 0831160522 ).

\section{References}

[1] W. Eerenstein, N. D. Mathur, and J. F. Scott, "Multiferroic and magnetoelectric materials," Nature, vol. 442, no. 7104, pp. 759-765, 2006.

[2] R. Ramesh and N. A. Spaldin, "Multiferroics: progress and prospects in thin films," Nature Materials, vol. 6, no. 1, pp. 2129, 2007.

[3] J. van Suchtelen, "Product properties: a new application of composite materials," Philips Research Reports, vol. 27, no. 1, pp. 28-37, 1972.
[4] C. W. Nan, "Magnetoelectric effect in composites of piezoelectric and piezomagnetic phases," Physical Review B, vol. 50, no. 9, pp. 6082-6088, 1994.

[5] C. W. Nan, M. I. Bichurin, S. X. Dong, D. Viehland, and G. Srinivasan, "Multiferroic magnetoelectric composites: historical perspective, status, and future directions," Journal of Applied Physics, vol. 103, no. 3, Article ID 031101, 35 pages, 2008.

[6] D. Chiba, M. Sawicki, Y. Nishitani, Y. Nakatani, F. Matsukura, and $\mathrm{H}$. Ohno, "Magnetization vector manipulation by electric fields," Nature, vol. 455, no. 7212, pp. 515-518, 2008.

[7] F. Zavaliche, T. Zhao, H. Zheng, et al., "Electrically assisted magnetic recording in multiferroic nanostructures," Nano Letters, vol. 7, no. 6, pp. 1586-1590, 2007.

[8] M. Liu, O. Obi, J. Lou, et al., "Giant electric field tuning of magnetic properties in multiferroic ferrite/ferroelectric heterostructures," Advanced Functional Materials, vol. 19, no. 11, pp. 1826-1831, 2009.

[9] N. Moutis, D. Suarez-Sandoval, and D. Niarchos, "Voltageinduced modification in magnetic coercivity of patterned $\mathrm{Co}_{50} \mathrm{Fe}_{50}$ thin film on piezoelectric substrate," Journal of Magnetism and Magnetic Materials, vol. 320, no. 6, pp. 1050$1055,2008$.

[10] S. Sahoo, S. Polisetty, C. G. Duan, S. S. Jaswal, E. Y. Tsymbal, and C. Binek, "Ferroelectric control of magnetism in $\mathrm{BaTiO}_{3} / \mathrm{Fe}$ heterostructures via interface strain coupling," Physical Review B, vol. 76, no. 9, Article ID 092108, 4 pages, 2007.

[11] R. E. Eitel, C. A. Randall, T. R. Shrout, and S. Park, "Preparation and characterization of high temperature perovskite ferroelectrics in the solid-solution $(1-\mathrm{x}) \mathrm{BiScO}_{3}-\mathrm{xPCTiO}_{3}$," Japanese Journal of Applied Physics, Part 1, vol. 41, no. 4A, pp. 2099-2104, 2002.

[12] T. T. Zou, X. H. Wang, W. Zhao, and L. T. Li, "Preparation and properties of fine-grain $(1-\mathrm{x}) \mathrm{BiScO}_{3}-\mathrm{xbTiO}_{3}$ ceramics by two-step sintering," Journal of the American Ceramic Society, vol. 91, no. 1, pp. 121-126, 2008.

[13] H. F. Tian, T. L. Qu, L. B. Luo, et al., "Strain induced magnetoelectric coupling between magnetite and $\mathrm{BaTiO}_{3}$," Applied Physics Letters, vol. 92, no. 6, Article ID 063507, 3 pages, 2008.

[14] L. Callegaro and E. Puppin, "Stress dependence of coercivity in Ni films: thin film to bulk transition," Applied Physics Letters, vol. 68, no. 9, pp. 1279-1281, 1996.

[15] P. K. Chakrabarti, F. Mazaleyrat, and L. K. Varga, "Soft magnetic properties of rapidly quenched pig-iron-based alloys," Journal of Magnetism and Magnetic Materials, vol. 254-255, pp. 447-449, 2003. 

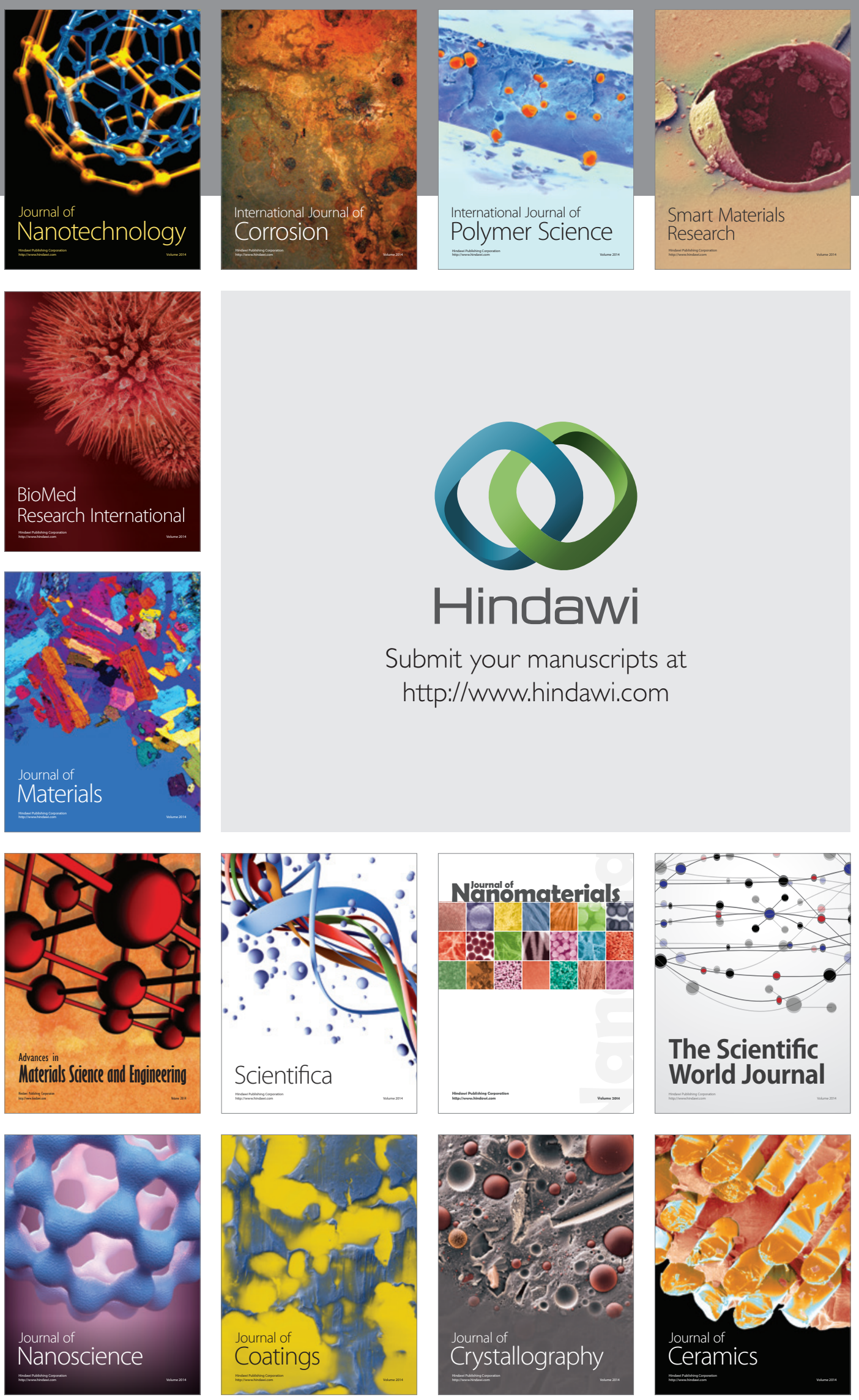

The Scientific World Journal

Submit your manuscripts at

http://www.hindawi.com

\section{World Journal}

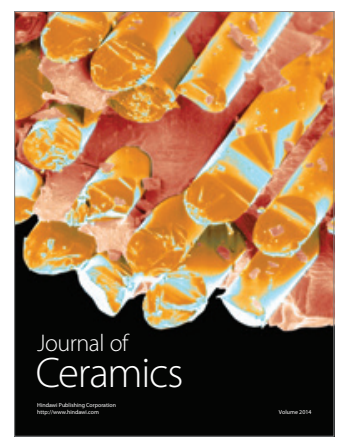

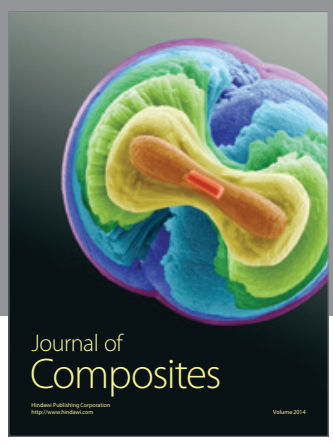
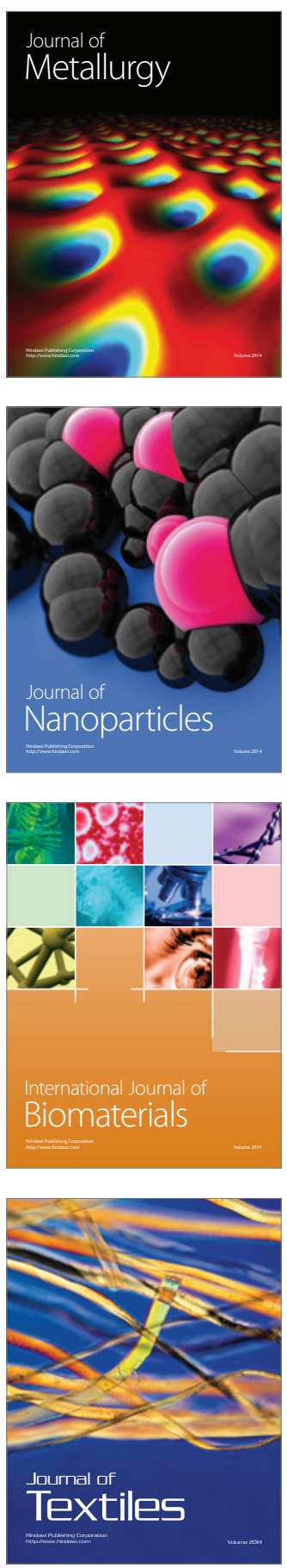